\title{
Nobel Lecture: One hundred years of light quanta*
}

\author{
Roy J. Glauber \\ Harvard University, Cambridge, Massachusetts 02138, USA
}

(Published 17 November 2006)

DOI: $10.1103 /$ RevModPhys.78.1267

It can't have escaped you, after so many recent reminders, that this year marks the one hundredth birthday of the light quantum. I thought I would tell you this morning a few things about its century long biography. Of course we have had light quanta on Earth for eons, in fact ever since the good Lord said "let there be quantum electrodynamics" - which is a modern translation, of course, from the biblical Aramaic. So in this talk I'll try to tell you what quantum optics is about, but there will hardly be enough time to tell you of the many new directions in which it has led us. Several of those are directions that we would scarcely have anticipated as all of this work started.

My own involvement in this subject began somewhere around the middle of the last century, but I would like to describe some of the background of the scene I entered at that point as a student. Let's begin, for a moment, even before the quantum theory was set in motion by Planck. It is important to recall some of the remarkable things that were found in the 19th century, thanks principally to the work of Thomas Young and Augustin Fresnel. They established within the first 20 years of the 19th century that light is a wave phenomenon, and that these waves, of whatever sort they might be, interpenetrate one another like waves on the surface of a pond. The wave displacements, in other words, add up algebraically. That's called superposition, and it was found thus that if you have two waves that remain lastingly in step with one another, they can add up constructively, and thereby reinforce one another in some places, or they can even oscillate oppositely to one another, and thereby cancel one another out locally. That would be what we call destructive interference.

Interference phenomena were very well understood by about 1820 . On the other hand, it wasn't at all understood what made up the underlying waves until the fundamental laws of electricity and magnetism were gathered together and augmented in a remarkable way by James Clerk Maxwell, who developed thereby the electrodynamics we know today. Maxwell's theory showed that light waves consist of oscillating electric and magnetic fields. The theory has been so perfect in describing the dynamics of electricity and magnetism over laboratory scale distances, that it has remained precisely intact.

\footnotetext{
*The 2005 Nobel Prize for Physics was shared by Roy J. Glauber, John L. Hall, and Theodor W. Hänsch. This lecture is the text of Dr. Glauber's address on the occasion of the award.
}

It has needed no fundamental additions in the years since the 1860s, apart from those concerning the quantum theory. It serves still, in fact, as the basis for the discussion and analysis of virtually all the optical instrumentation we have ever developed. That overwhelming and continuing success may eventually have led to a certain complacency. It seemed to imply that the field of optics, by the middle of the 20th century, scarcely needed to take any notice of the granular nature of light. Studying the behavior of light quanta was then left to the atomic and elementary particle physicists-whose interests were largely directed toward other phenomena.

The story of the quantum theory, of course, really begins with Max Planck. Planck in 1900 was confronted with many measurements of the spectral distribution of the thermal radiation that is given off by a hot object. It was known that under ideally defined conditions, that is, for complete (or black) absorbers and correspondingly perfect emitters this is a unique radiation distribution. The intensities of its color distribution, under such ideal conditions, should depend only on temperature and not at all on the character of the materials that are doing the radiating. That defines the so-called blackbody distribution. Planck, following others, tried finding a formula that expresses the shape of that blackbody color spectrum. Something of its shape was known at low frequencies, and there was a good guess present for its shape at high frequencies.

The remarkable thing that Planck did first was simply to devise an empirical formula that interpolates between those two extremes. It was a relatively simple formula and it involved one constant which he had to adjust in order to fit the data at a single temperature. Then having done that, he found his formula worked at other temperatures. He presented the formula to the German Physical Society on October 19, 1900 (Planck, 1900a) and it turned out to be successful in describing still newer data. Within a few weeks the formula seemed to be established as a uniquely correct expression for the spectral distribution of thermal radiation.

The next question obviously was: Did this formula have a logical derivation of any sort? There Planck, who was a sophisticated theorist, ran into a bit of trouble. First of all he understood from his thermodynamic background that he could base his discussion on nearly any model of matter, however oversimplified it might be, as long as it absorbed and emitted light efficiently. So he based his model on the mechanical system he understood best, a collection of one-dimensional harmonic os- 
cillators, each of them oscillating rather like a weight at the end of a spring. They had to be electrically charged. He knew from Maxwell exactly how these charged oscillators interact with the electromagnetic field. They both radiate and absorb in a way he could calculate. So then he ought to be able to find the equilibrium between these oscillators and the radiation field, which acted as a kind of thermal reservoir-and which he never made any claim to discuss in detail.

He found that he could not secure a derivation for his magic formula for the radiation distribution unless he made an assumption which, from a philosophical standpoint, he found all but unacceptable. The assumption was that the harmonic oscillators he was discussing had to possess energies that were distributed, not as the continuous variables one expected, but confined instead to discrete and regularly spaced values. The oscillators of frequency $\nu$ would have to be restricted to energy values that were integer multiples, i.e., $n$-fold multiples (with $n=0,1,2,3, \ldots)$ of something he called the quantum of energy, $h \nu$.

That number $h$ was, in effect, the single number that he had to introduce in order to fit his magic formula to the observed data at a single temperature. So he was saying, in effect, that these hypothetical harmonic oscillators representing a simplified image of matter could have only a sequence amounting to a "ladder" of energy states. That assumption permits us to see immediately why the thermal radiation distribution must fall off rapidly with rising frequency. The energy steps between the oscillator states grow larger, according to his assumption, as the frequency rises, but thermal excitation energies, on the other hand, are quite restricted in magnitude at any fixed temperature. High frequency oscillators at thermal equilibrium would never even reach the first step of excitation. Hence there tends to be very little high frequency radiation present at thermal equilibrium. Planck presented this revolutionary suggestion (Planck, 1900b) to the Physical Society on December 14, 1900, although he could scarcely believe it himself.

The next great innovation came in 1905 from the young Albert Einstein, employed still at the Bern Patent Office. Einstein first observed that Planck's formula for the entropy of the radiation distribution, when he examined its high frequency contributions, looked like the entropy of a perfect gas of free particles of energy $h \nu$. That was a suggestion that light itself might be discrete in nature, but hardly a conclusive one.

To reach a stronger conclusion he turned to an examination of the photoelectric effect, which had first been observed in 1887 by Heinrich Hertz. Shining monochromatic light on metal surfaces drives electrons out of the metals, but only if the frequency of the light exceeds a certain threshold value characteristic of each metal. It would have been most reasonable to expect that as one shines more intense light on those metals the electrons would come out faster, that is, with higher velocities in response to the stronger oscillating electric fields-but they don't. They come out always with the same velocities, provided that the incident light is of a frequency higher than the threshold frequency. If it were below that frequency there would be no photoelectrons at all.

The only response that the metals make to increasing the intensity of light lies in producing more photoelectrons. Einstein had a naively simple explanation for that (Einstein, 1905). The light itself, he assumed, consists of localized energy packets and each possesses one quantum of energy. When light strikes the metal, each packet is absorbed by a single electron. That electron then flies off with a unique energy, an energy which is just the packet energy $h \nu$ minus whatever energy the electron needs to expend in order to escape the metal.

It took until about 1914-1916 to secure an adequate verification of Einstein's law for the energies of the photoelectrons. When Millikan succeeded in doing that, it seemed clear that Einstein was right, and that light does indeed consist of quantized energy packets. It was thus Einstein who fathered the light quantum, in one of the several seminal papers he wrote in the year 1905.

To follow the history a bit further, Einstein began to realize in 1909 that his energy packets would have a momentum which, according to Maxwell, should be their energy divided by the velocity of light. These presumably localized packets would have to be emitted in single directions if they were to remain localized, or to constitute "Nadelstrahlung" (needle radiation), very different in behavior from the broadly continuous angular distribution of radiation that would spread from harmonic oscillators according to the Maxwell theory. A random distribution of these needle radiations would look appropriately continuous, but what was disturbing about that was the randomness with which these needle radiations would have to appear. That was evidently the first of the random variables in the quantum theory that began disturbing Einstein and kept nettling him for the rest of his life.

In 1916 Einstein found another and very much more congenial way of deriving Planck's distribution by discussing the rate at which atoms radiate. Very little was known about atoms at that stage save that they must be capable of absorbing and giving off radiations. An atom lodged in a radiation field would surely have its constituent charges shaken by the field oscillations, and that shaking could lead either to the absorption of radiation or to the emission of still more radiation. Those were the processes of absorption or emission induced by the prior presence of radiation. But Einstein found that thermal equilibrium between matter and radiation could only be reached if, in addition to these induced processes, there exists also a spontaneous process, one in which an excited atom emits radiation even in the absence of any prior radiation field. It would be analogous to radioactive decays discovered by Rutherford. The rates at which these processes take place were governed by Einstein's famous $B$ and $A$ coefficients, respectively. The existence of spontaneous radiation turned out to be an important guide to the construction of quantum electrodynamics.

Some doubts about the quantized nature of light inevitably persisted, but many of them were dispelled by 
Compton's discovery in 1922 that x-ray quanta are scattered by electrons according to the same rules as govern the collisions of billiard balls. They both obey the conservation rules for energy and momentum in much the same way. It became clear that the particle picture of light quanta, whatever were the dilemmas that accompanied it, was here to stay.

The next dramatic developments of the quantum theory, of course, took place between the years 1924 and 1926. They had the effect of ascribing to material particles such as electrons much of the same wavelike behavior as had long since been understood to characterize light. In those developments de Broglie, Heisenberg, Schrödinger, and others accomplished literal miracles in explaining the structure of atoms. But, however much this invention of modern quantum mechanics succeeded in laying the groundwork for a more general theory of the structure of matter, it seemed at first to have little new bearing on the understanding of electromagnetic phenomena. The spontaneous emission of light persisted as an outstanding puzzle.

Thus there remained a period of a couple of years more in which we described radiation processes in terms that have usually been called "semiclassical." Now the term "classical" is an interesting one-because, as you know, every field of study has its classics. Many of the classics that we are familiar with go back two or three thousand years in history. Some are less old, but all share an antique, if not ancient, character. In physics we are a great deal more precise, as well as contemporary. Anything that we understood or could have understood prior to the date of Planck's paper, December 14, 1900, is to us "classical." Those understandings are our classics. It is the introduction of Planck's constant that marks the transition from the classical era to our modern one.

The true "semiclassical era," on the other hand, lasted only about two years. It ended formally with the discovery by Paul Dirac $(1927,1927)$ that one must treat the vacuum, that is to say, empty space, as a dynamical system. The energy distributed through space in an electromagnetic field had been shown by Maxwell to be a quadratic expression in the electric and magnetic field strengths. Those quadratic expressions are formally identical in their structure to the mathematical expressions for the energies of mechanical harmonic oscillators. Dirac observed that even though there may not seem to be any organized fields present in the vacuum those mathematically defined oscillators that described the field energy would make contributions that could not be overlooked. The quantum mechanical nature of the oscillators would add an important but hitherto neglected correction to the argument of Planck.

Planck had said the energies of harmonic oscillators are restricted to values $n$ times the quantum energy $h \nu$ and the fully developed quantum mechanics had shown in fact that those energies are not $n h \nu$ but $\left(n+\frac{1}{2}\right) h \nu$. All of the intervals between energy levels remained unchanged, but the quantum mechanical uncertainty principle required that additional $\frac{1}{2} h \nu$ to be present. We can never have a harmonic oscillator completely empty of energy because that would require its position coordinate and its momentum simultaneously to have the precise values zero.

So, according to Dirac, the electromagnetic field is made up of field amplitudes that can oscillate harmonically. But these amplitudes, because of the ever-present half quantum of energy $\frac{1}{2} h \nu$, can never be permanently at rest. They must always have their fundamental excitations, the so-called "zero-point fluctuations" going on. The vacuum then is an active dynamical system. It is not empty. It is forever buzzing with weak electromagnetic fields. They are part of the ground state of emptiness. We can withdraw no energy at all from those fluctuating electromagnetic fields. We have to regard them nonetheless as real and present even though we are denied any way of perceiving them directly.

An immediate consequence of this picture was the unification of the notions of spontaneous and induced emission. Spontaneous emission is emission induced by those zero-point oscillations of the electromagnetic field. Furthermore it furnishes, in a sense, an indirect way of perceiving the zero-point fluctuations by amplifying them. Quantum amplifiers tend to generate background noise that consists of radiation induced by those vacuum fluctuations.

It is worth pointing out a small shift in terminology that took place in the late 1920s. Once material particles were found to exhibit some of the wavelike behavior of light quanta, it seemed appropriate to acknowledge that the light quanta themselves might be elementary particles, and to call them "photons" as suggested by G. N. Lewis in 1926. They seemed every bit as discrete as material particles, even if their existence was more transitory, and they were at times freely created or annihilated.

The countless optical experiments that had been performed by the middle of the 20th century were in one or another way based on detecting only the intensity of light. It may even have seemed there wasn't anything else worth measuring. Furthermore, those measurements were generally made with steady light beams traversing passive media. It proved quite easy therefore to describe those measurements in simple and essentially classical terms. A characteristic first mathematical step was to split the expression for the oscillating electric field $E$ into two complex conjugate terms:

$$
\begin{aligned}
& E=E^{(+)}+E^{(-)}, \\
& E^{(-)}=\left(E^{(+)}\right)^{*},
\end{aligned}
$$

with the understanding that $E^{(+)}$contains only positive frequency terms, i.e., those varying as $e^{-i \omega t}$ for all $\omega>0$, and $E^{(-)}$contains only negative frequency terms $e^{i \omega t}$. This is a separation familiar to electrical engineers and motivated entirely by the mathematical convenience of dealing with exponential functions. It has no physical motivation in the context of classical theory, since the two complex fields $E^{( \pm)}$are physically equivalent. They 


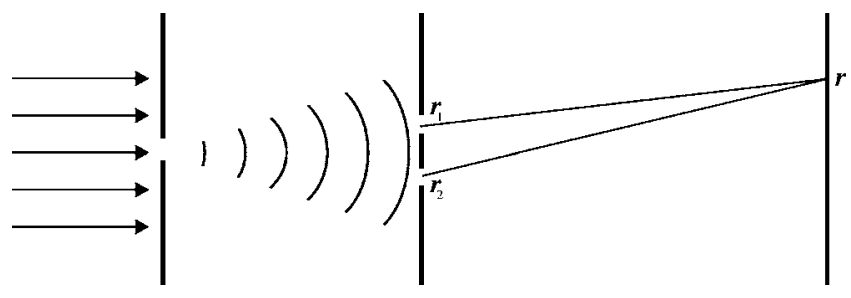

FIG. 1. Young's experiment. Light passing through a pinhole in the first screen falls on two closely spaced pinholes in a second screen. The superposition of the waves radiated by those pinholes at $\boldsymbol{r}_{1}$ and $\boldsymbol{r}_{2}$ leads to interference fringes seen at points $\boldsymbol{r}$ on the third screen.

furnish identical descriptions of classical theory.

Each of the fields $E^{( \pm)}(\boldsymbol{r} t)$ depends in general on both the space coordinate $\boldsymbol{r}$ and time $t$. The instantaneous field intensity at $\boldsymbol{r}, t$ would then be

$$
\left|E^{(+)}(\boldsymbol{r}, t)\right|^{2}=E^{(-)}(\boldsymbol{r}, t) E^{(+)}(\boldsymbol{r}, t) .
$$

In practice it was always an average intensity that was measured, usually a time average.

The truly ingenious element of many optical experiments, going all the way back to Young's double-pinhole experiment, was the means their design afforded to superpose the fields arriving at different space-time points before the intensity observations were made. Thus in Young's experiment, shown in Fig. 1, light penetrating a single pinhole in the first screen passes through two pinholes in the second screen and then is detected as it falls on a third screen. The field $E^{(+)}(\boldsymbol{r} t)$ at any point on the latter screen is the superposition of two waves radiated from the two prior pinholes with a slight difference in their arrival times at the third screen, due to the slightly different distances they have to travel.

If we wanted to discuss the resulting light intensities in detail, we would find it most convenient to do that in terms of a field correlation function which we shall define as

$$
G^{(1)}\left(\boldsymbol{r}_{1} t_{1}, \boldsymbol{r}_{2} t_{2}\right)=\left\langle E^{(-)}\left(\boldsymbol{r}_{1} t_{1}\right) E^{(+)}\left(\boldsymbol{r}_{2} t_{2}\right)\right\rangle .
$$

This is a complex-valued function that depends, in general, on both space-time points $\boldsymbol{r}_{1} t_{1}$ and $\boldsymbol{r}_{2} t_{2}$. The angular brackets $\langle\cdots\rangle$ indicate that an average value is somehow taken, as we have noted. The average intensity of the field at the single point $\boldsymbol{r} t$ is then just $G^{(1)}(\boldsymbol{r} t, \boldsymbol{r} t)$.

If the field $E^{(+)}(\boldsymbol{r} t)$ at any point on the third screen is given by the sum of two fields, i.e., proportional to $E^{(+)}\left(\boldsymbol{r}_{1} t_{1}\right)+E^{(+)}\left(\boldsymbol{r}_{2} t_{2}\right)$, then it is easy to see that the average intensity at $\boldsymbol{r} t$ on the screen is given by a sum of four correlation functions,

$$
\begin{aligned}
& G^{(1)}\left(\boldsymbol{r}_{1} t_{1} \boldsymbol{r}_{1} t_{1}\right)+G^{(1)}\left(\boldsymbol{r}_{2} t_{2} \boldsymbol{r}_{2} t_{2}\right) \\
& \quad+G^{(1)}\left(\boldsymbol{r}_{1} t_{1} \boldsymbol{r}_{2} t_{2}\right)+G^{(1)}\left(\boldsymbol{r}_{2} t_{2} \boldsymbol{r}_{1} t_{1}\right) .
\end{aligned}
$$

The first two of these terms are the separate contributions of the two pinholes in the second screen, that is, the intensities they would contribute individually if each alone were present. Those smooth intensity distributions are supplemented, however, by the latter two terms of the sum, which represent the characteristic interference effect of the superposed waves. They are the terms that lead to the intensity fringes observed by Young.

Intensity fringes of that sort assume the greatest possible contrast or visibility when the cross-correlation terms like $G^{(1)}\left(\boldsymbol{r}_{1} t_{1} \boldsymbol{r}_{2} t_{2}\right)$ are as large in magnitude as possible. But there is a simple limitation imposed on the magnitude of such correlations by a familiar inequality. There is a formal sense in which cross-correlation functions like $G^{(1)}\left(\boldsymbol{r}_{1} t_{1} \boldsymbol{r}_{2} t_{2}\right)$ are analogous to the scalar products of two vectors and are thus subject to a Schwarz inequality. The squared absolute value of that correlation function can then never exceed the product of the two intensities. If we let $x$ abbreviate a coordinate pair $\boldsymbol{r}, t$, we must have

$$
\left|G^{(1)}\left(x_{1} x_{2}\right)\right|^{2} \leqslant G^{(1)}\left(x_{1} x_{1}\right) G^{(1)}\left(x_{2} x_{2}\right) .
$$

The upper bound to the cross-correlation is attained if we have

$$
\left|G^{(1)}\left(x_{1} x_{2}\right)\right|^{2}=G^{(1)}\left(x_{1} x_{1}\right) G^{(1)}\left(x_{2} x_{2}\right),
$$

and with it we achieve maximum fringe contrast. We shall then speak of the fields at $x_{1}$ and $x_{2}$ as being optically coherent with one another. That is the definition of relative coherence that optics has traditionally used (Born and Wolf, 1959).

There is another way of stating the condition for optical coherence that is also quite useful, particularly when we are discussing coherence at pairs of points extending over some specified region in space-time. Let us assume that it is possible to find a positive frequency field $\mathcal{E}(\boldsymbol{r} t)$ satisfying the appropriate Maxwell equations and such that the correlation function (4) factorizes into the form

$$
G^{(1)}\left(\boldsymbol{r}_{1} t_{1}, \boldsymbol{r}_{2} t_{2}\right)=\mathcal{E}^{*}\left(\boldsymbol{r}_{1} t_{1}\right) \mathcal{E}\left(\boldsymbol{r}_{2} t_{2}\right) .
$$

While the necessity of this factorization property requires a bit of proof (Titulaer and Glauber, 1965, 1966) it is at least clear that it does bring about the optical coherence that we have defined by means of the upper bound in the inequality (6) since in that case we have

$$
\left|G^{(1)}\left(\boldsymbol{r}_{1} t_{1} \boldsymbol{r}_{2} t_{2}\right)\right|^{2}=\left|E\left(\boldsymbol{r}_{1} t_{1}\right)\right|^{2}\left|E\left(\boldsymbol{r}_{2} t_{2}\right)\right|^{2} .
$$

In the quantum theory, physical variables such as $E^{( \pm)}(\boldsymbol{r} t)$ are associated not with simple complex numbers but with operators on the Hilbert-space vectors |\rangle that represent the state of the system, which in the present case is the electromagnetic field. Multiplication of the operators $E^{(+)}\left(\boldsymbol{r}_{1} t_{1}\right)$ and $E^{(-)}\left(\boldsymbol{r}_{2} t_{2}\right)$ is not in general commutative, and the two operators can be demonstrated to act in altogether different ways on the vectors |\rangle that represent the state of the field. The operator $E^{(+)}$, in particular, can be shown to be an annihilation operator. It lowers by 1 the number of quanta present in the field. Applied to an $n$-photon state, $|n\rangle$, it reduces it to an $n-1$ photon state, $|n-1\rangle$. Further applications of $E^{(+)}(\boldsymbol{r} t)$ keep reducing the number of quanta present still 
further, but the sequence must end with the $n=0$ or vacuum state, $|\mathrm{vac}\rangle$, in which there are no quanta left. In that state we must finally have

$$
E^{(+)}(\boldsymbol{r} t)|\mathrm{vac}\rangle=0 \text {. }
$$

The operator adjoint to $E^{(+)}$, which is $E^{(-)}$, must have the property of raising an $n$-photon state to an $n+1$-photon state, so we may be sure, for example, that the state $E^{(-)}(\boldsymbol{r} t)|\mathrm{vac}\rangle$ is a one-photon state. Since the vacuum state cannot be reached by raising the number of photons, we must also require the relation

$$
\langle\operatorname{vac}| E^{(-)}(\boldsymbol{r} t)=0,
$$

which is adjoint to Eq. (10).

The results of quantum measurements often depend on the way in which the measurements are carried out. The most useful and informative ways of discussing such experiments are usually those based on the physics of the measurement process itself. To discuss measurements of the intensity of light then we should understand the functioning of the device that detects or counts photons.

Such devices generally work by absorbing quanta and registering each such absorption process, for example, by the detection of an emitted photoelectron. We need not go into any of the details of the photoabsorption process to see the general nature of the expression for the photon counting probability. All we need to assume is that our idealized detector at the point $\boldsymbol{r}$ has negligibly small size and has a photoabsorption probability that is independent of frequency so that it can be regarded as probing the field at a well defined time $t$. Then if the field makes a transition from an initial state $|i\rangle$ to a final state $|f\rangle$ in which there is one photon fewer, the probability amplitude for that particular transition is given by the scalar product_or matrix element

$$
\left\langle f\left|E^{(+)}(\boldsymbol{r} t)\right| i\right\rangle .
$$

To find the total transition probability we must find the squared modulus of this amplitude and sum it over the complete set of possible final states $|f\rangle$ for the field. The expression for the completeness of the set of states $|f\rangle$ is

$$
\sum_{f}|f\rangle\langle f|=1
$$

so that we then have a total transition probability proportional to

$$
\begin{aligned}
\sum_{f}\left|\left\langle f\left|E^{(+)}(\boldsymbol{r} t)\right| i\right\rangle\right|^{2} & =\sum_{f}\left\langle i\left|E^{(-)}(\boldsymbol{r} t)\right| f\right\rangle\left\langle f\left|E^{(+)}(\boldsymbol{r} t)\right| i\right\rangle \\
& =\left\langle i\left|E^{(-)}(\boldsymbol{r} t) E^{(+)}(\boldsymbol{r} t)\right| i\right\rangle .
\end{aligned}
$$

It is worth repeating here that in the quantum theory the fields $E^{( \pm)}$are noncommuting operators rather than simple numbers. Thus one could not reverse their ordering in the expression (13) while preserving its meaning. In the classical theory we discussed earlier $E^{(+)}$and $E^{(-)}$ are simple numbers that convey equivalent information. There is no physical distinction between photoabsorption and emission since there are no classical photons.
The fact that the quantum energy $h \nu$ vanishes for $h \rightarrow 0$ removes any distinction between positive and negative values of the frequency variable.

The initial state of the field in our photon counting experiment depends, of course, on the output of whatever light source we use, and very few sources produce pure quantum states of any sort. We must thus regard the state $|i\rangle$ as depending in general on some set of random and uncontrollable parameters characteristic of the source. The counting statistics we actually measure then may vary from one repetition of the experiment to another. The figure we would quote must be regarded as the average taken over these repetitions. The neatest way of specifying the random properties of the state $|i\rangle$ is to define what von Neumann called the density operator

$$
\rho=\{|i\rangle\langle i|\}_{\mathrm{av}},
$$

which is the statistical average of the outer product of the vector $|i\rangle$ with itself. Expression (14) permits us to write the average of the counting probability as

$$
\left.\left\{\left\langle i\left|E^{(-)}(\boldsymbol{r} t) E^{(+)}(\boldsymbol{r} t)\right| i\right\rangle\right\}_{\mathrm{av}}=\operatorname{Tr}\left\{\rho E^{(-)}(\boldsymbol{r} t) E^{(+)} \boldsymbol{r} t\right)\right\} .
$$

Interference experiments like those of Young and Michelson, as we have noted earlier, often proceed by measuring the intensities of linear combinations of the fields characteristic of two different space-time points. To find the counting probability in a field like $E^{(+)}\left(\boldsymbol{r}_{1} t_{1}\right)$ $+E^{(+)}\left(\boldsymbol{r}_{2} t_{2}\right)$, for example, we will need to know expressions like that of Eq. (15) but with two different spacetime arguments $\boldsymbol{r}_{1} t_{1}$ and $\boldsymbol{r}_{2} t_{2}$. It is convenient then to define the quantum theoretical form of the correlation functions (4) as

$$
G^{(1)}\left(\boldsymbol{r}_{1} t_{1} \boldsymbol{r}_{2} t_{2}\right)=\operatorname{Tr}\left\{\rho E^{(-)}\left(\boldsymbol{r}_{1} t_{1}\right) E^{(+)}\left(\boldsymbol{r}_{2} t_{2}\right)\right\} .
$$

This function has the same scalar product structure as the classical function (4) and can be shown likewise to obey the inequality (6). Once again we can take the upper bound of the modulus of this cross-correlation function or equivalently the factorization condition (8) to define optical coherence.

It is worth noting at this point that optical experiments aimed at achieving a high degree of coherence have almost always accomplished it by using the most nearly monochromatic light attainable. The reason for that is made clear by the factorization condition (8). These experiments were always based on steady or statistically stationary light sources. What we mean by a steady state is that the function $G^{(1)}$ with two different time arguments, $t_{1}$ and $t_{2}$, can in fact only depend on their difference $t_{1}-t_{2}$. Optical coherence then requires

$$
G^{(1)}\left(t_{1}-t_{2}\right)=\mathcal{E}^{*}\left(t_{1}\right) \mathcal{E}\left(t_{2}\right) .
$$

The only possible solution of such a functional equation for the function $E(t)$ is one that oscillates with a single positive frequency. The requirement of monochromaticity thus follows from the limitation to steady sources. The factorization condition (8), on the other hand, defines optical coherence more generally for nonsteady sources as well. 


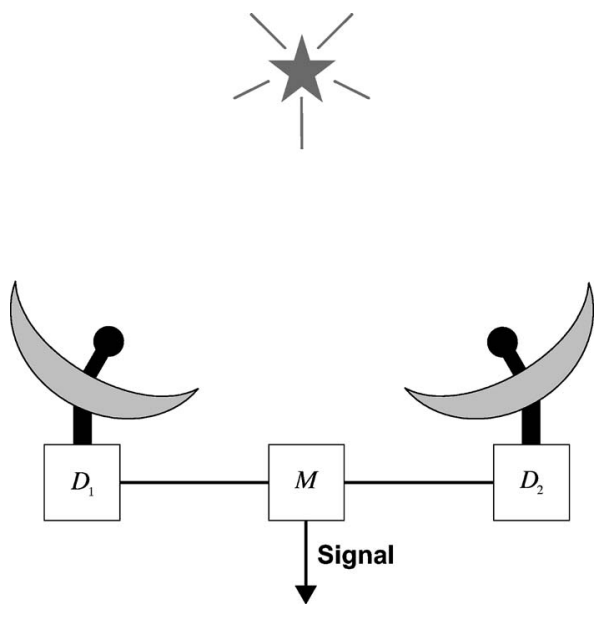

FIG. 2. The intensity interferometry scheme of Hanbury Brown and Twiss. Radio-frequency waves are received and detected at two antennas. The filtered low-frequency signals that result are sent to a device that furnishes an output proportional to their product.

Although the energies of visible light quanta are quite small on the everyday scale, techniques for detecting them individually have existed for many decades. The simplest methods are based on the photoelectric effect and the use of electron photomultipliers to produce well defined current pulses. The possibility of detecting individual quanta raises interesting questions concerning their statistical distributions, distributions that should in principle be quite accessible to measurement. We might imagine, for example, putting a quantum counter in a given light beam and asking for the distribution of time intervals between successive counts. Statistical problems of that sort were never, to my knowledge, addressed until the importance of quantum correlations began to become clear in the 1950s. Until that time virtually all optical experiments measured only average intensities or quantum counting rates, and the correlation function $G^{(1)}$ was all we needed to describe them. It was in that decade, however, that several new sorts of experiments requiring a more general approach were begun. That period seemed to mark the beginning of quantum optics as a relatively new or rejuvenated field.

In the experiment I found most interesting, R. Hanbury Brown and R. Q. Twiss developed a new form of interferometry (Hanbury Brown, 1954). They were interested at first in measuring the angular sizes of radio wave sources in the sky and found they could accomplish that by using two antennas, as shown in Fig. 2, with a detector attached to each of them to remove the highfrequency oscillations of the field. The noisy lowfrequency signals that were left were then sent to a central device that multiplied them together and recorded their time-averaged values. Each of the two detectors then was producing an output proportional to the square of the incident field, and the central device was recording a quantity that was quartic in the field strengths.

It is easy to show, by using classical expressions for the field strengths, that the quartic expression contains a

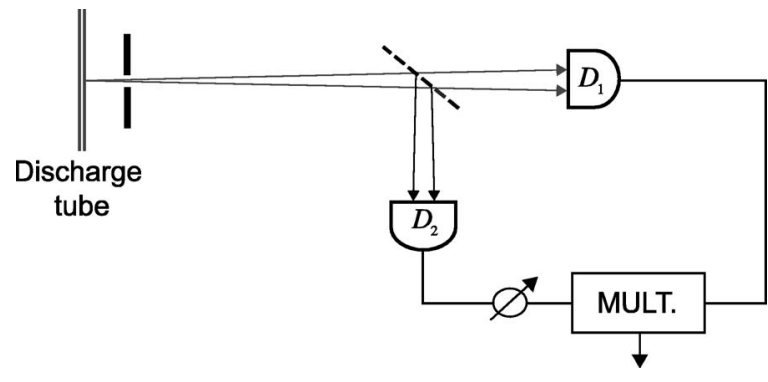

FIG. 3. The Hanbury Brown-Twiss photon correlation experiment. Light from an extremely monochromatic discharge tube falls on a half-silvered mirror which sends the split beam to two separate photodetectors. The random photocurrents from the two detectors are multiplied together and then averaged. The variable time delay indicated is actually achieved by varying the distance of the detector $D_{2}$ from the mirror.

measurable interference term, and by exploiting it Hanbury Brown and Twiss did measure the angular sizes of many radio sources. They then asked themselves whether they couldn't perform the same sort of "intensity interferometry" with visible light, and thereby measure the angular diameters of visible stars. Although it seemed altogether logical that they could do that, the interference effect would have to involve the detection of pairs of photons and they were evidently inhibited in imagining the required interference effect by a statement Dirac makes in the first chapter of his famous textbook on quantum mechanics (Dirac, 1958). In it he is discussing why one sees intensity fringes in the Michelson interferometer, and says in ringingly clear terms, "Each photon then interferes only with itself. Interference between two different photons never occurs."

It is worth recalling at this point that interference simply means that the probability amplitudes for alternative and indistinguishable histories must be added together algebraically. It is not the photons that interfere physically, it is their probability amplitudes that interfereand probability amplitudes can be defined equally well for arbitrary numbers of photons.

Evidently Hanbury Brown and Twiss remained uncertain on this point and undertook an experiment (Hanbury Brown and Twiss, 1956, 1957a, 1957b) to determine whether pairs of photons can indeed interfere. Their experimental arrangement is shown in Fig. 3. The light source is an extremely monochromatic discharge tube. The light from that source is collimated and sent to a half-silvered mirror which sends the separated beams to two separate photodetectors. The more or less random output signals of those two detectors are multiplied together, as they were in the radio-frequency experiments, and then averaged. The resulting averages showed a slight tendency for both of the photodetectors to register photons simultaneously (Fig. 4). The effect could be removed by displacing one of the counters and thus introducing an effective time delay between them. The coincidence effect thus observed was greatly weakened by the poor time resolution of the detectors, but it raised considerable surprise nonetheless. The observation of 


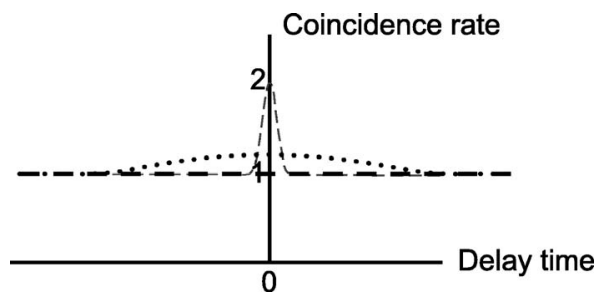

FIG. 4. The photon coincidence rate measured rises slightly above the constant background of accidental coincidences for sufficiently small time delays. The observed rise was actually weakened in magnitude and extended over longer time delays by the relatively slow response of the photodetectors. With ideal detectors it would take the more sharply peaked form shown.

temporal correlations between photons in a steady beam was something altogether new. The experiment has been repeated several times, with better resolution, and the correlation effect has emerged in each case more clearly (Rebka and Pound, 1957; Scarl, 1968).

The correlation effect was enough of a surprise to call for a clear explanation. The closest it came to that was a clever argument (Purcell, 1956) by Purcell who used the semiclassical form of the radiation theory in conjunction with a formula for the relaxation time of radiofrequency noise developed in wartime radar studies. It seemed to indicate that the photon correlation time would be increased by just using a more monochromatic light source.

The late 1950s were, of course, the time in which the laser was being developed, but it was not until 1960 that the helium-neon laser (Javan et al., 1961) was on the scene with its extremely monochromatic and stable beams. The question then arose: What are the correlations of the photons in a laser beam? Would they extend, as one might guess, over much longer time intervals as the beam became more monochromatic? I puzzled over the question for some time, I must admit, since it seemed to me, even without any detailed theory of the laser mechanism, that there would not be any such extended correlation.

The oscillating electric current that radiates light in a laser tube is not a current of free charges. It is a polarization current of bound charges oscillating in a direction perpendicular to the axis of the tube (Fig. 5). If it is sufficiently strong it can be regarded as a predetermined classical current, one that suffers negligible recoil when individual photons are emitted. Such currents, I knew

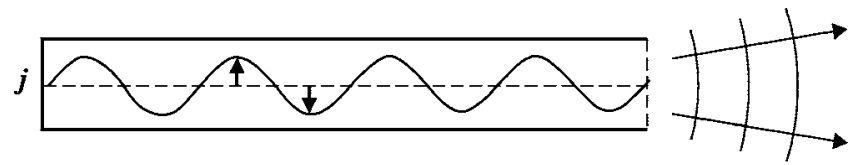

Strong oscillating polarization current $j=\frac{\partial P}{\partial t}$

FIG. 5. Schematic picture of a gas laser. The standing light wave in the discharge tube generates an intense transverse polarization current in the gas. Its oscillation sustains the standing wave and generates the radiated beam.
(Glauber, 1951), emitted Poisson distributions of photons, which indicated clearly that the photons were statistically independent of one another. It seemed then that a laser beam would show no Hanbury Brown-Twiss photon correlations at all.

How then would one describe the delayedcoincidence counting measurement of Hanbury Brown and Twiss? If the two photon counters are sensitive at the space-time points $\boldsymbol{r}_{1} t_{1}$ and $\boldsymbol{r}_{2} t_{2}$, we will need to make use of the annihilation operators $E^{(+)}\left(\boldsymbol{r}_{1} t_{1}\right)$ and $E^{(+)}\left(\boldsymbol{r}_{2} t_{2}\right)$ (which do, in fact commute). The amplitude for the field to go from the state $|i\rangle$ to a state $|f\rangle$ with two quanta fewer is

$$
\left\langle f\left|E^{(+)}\left(\boldsymbol{r}_{2} t_{2}\right) E^{(+)}\left(\boldsymbol{r}_{1} t_{1}\right)\right| i\right\rangle .
$$

When this expression is squared, summed over final states $|f\rangle$, and averaged over the initial states $|i\rangle$ we have a new kind of correlation function that we can write as

$$
\begin{aligned}
G^{(2)}\left(\boldsymbol{r}_{1} t_{1} \boldsymbol{r}_{2} t_{2} \boldsymbol{r}_{2} t_{2} \boldsymbol{r}_{1} t_{1}\right)= & \operatorname{Tr}\left\{\rho E^{(-)}\left(\boldsymbol{r}_{1} t_{1}\right) E^{(-)}\left(\boldsymbol{r}_{2} t_{2}\right)\right. \\
& \left.\times E^{(+)}\left(\boldsymbol{r}_{2} t_{2}\right) E^{(+)}\left(\boldsymbol{r}_{1} t_{1}\right)\right\} .
\end{aligned}
$$

This is a special case of a somewhat more general second-order correlation function that we can write (with the abbreviation $x_{j}=\boldsymbol{r}_{j} t_{j}$ ) as

$$
G^{(2)}\left(x_{1} x_{2} x_{3} x_{4}\right)=\operatorname{Tr}\left\{\rho E^{(-)}\left(x_{1}\right) E^{(-)}\left(x_{2}\right) E^{(+)}\left(x_{3}\right) E^{(+)}\left(x_{4}\right)\right\} .
$$

Now Hanbury Brown and Twiss had seen to it that the beams falling on their two detectors were as coherent as possible in the usual optical sense. The function $G^{(1)}$ should thus have satisfied the factorization condition (8), but that statement doesn't at all imply any corresponding factorization property of the functions $G^{(2)}$ given by Eq. (19) or (20).

We are free to define a kind of second-order coherence by requiring a parallel factorization of $G^{(2)}$,

$$
G^{(2)}\left(x_{1} x_{2} x_{3} x_{4}\right)=\mathcal{E}^{*}\left(x_{1}\right) \mathcal{E}^{*}\left(x_{2}\right) \mathcal{E}\left(x_{3}\right) \mathcal{E}\left(x_{4}\right),
$$

and the definition can be a useful one even though the Hanbury Brown-Twiss correlation assures us that no such factorization is present in their experiment. If it were present, the coincidence rate according to Eq. (21) would be proportional to

$$
G^{(2)}\left(x_{1} x_{2} x_{2} x_{1}\right)=G^{(1)}\left(x_{1} x_{1}\right) G^{(1)}\left(x_{2} x_{2}\right),
$$

that is, to the product of the two average intensities measured separately-and that is what was not found. Ordinary light beams, that is, light from ordinary sources, even extremely monochromatic ones as in the Hanbury Brown-Twiss experiment, do not have any such second-order coherence.

We can go on defining still higher-order forms of coherence by defining $n$ th-order correlation functions $G^{(n)}$ that depend on $2 n$ space-time coordinates. The usefulness of such functions may not be clear since carrying out the $n$-fold delayed coincidence counting experiments that measure them would be quite difficult in practice. It is nonetheless useful to discuss such functions since they 
do turn out to play an essential role in most calculations of the statistical distributions of photons. If we turn on a photon counter for any given length of time, for example, the number of photons it records will be a random integer. Repeating the experiment many times will lead us to a distribution function for that number. To predict those distributions (Glauber, 1965) we need, in general, to know the correlation functions $G^{(n)}$ of arbitrary orders.

Once we are defining higher-order forms of coherence, it is worth asking whether we can find fields that lead to factorization of the complete set of correlation functions $G^{(n)}$. If so, we could speak of those as possessing full coherence. Now, are there any such states of the field? In fact there are lots of them, and some can describe precisely the fields generated by predetermined classical current distributions. These fields have the remarkable property that annihilating a single quantum in them by means of the operator $E^{(+)}$leaves the field essentially unchanged. It just multiplies the state vector by an ordinary number. That is a relation we can write as

$$
E^{(+)}(\boldsymbol{r} t)|\rangle=\mathcal{E}(\boldsymbol{r} t)|\rangle,
$$

where $\mathcal{E}(\boldsymbol{r} t)$ is a positive frequency function of the spacetime point $\boldsymbol{r} t$. It is immediately clear that such states must have indefinite numbers of quanta present. Only in that way can they remain unchanged when one quantum is removed. This remarkable relation does in fact hold for all of the quantum states radiated by a classical current distribution, and in that case the function $\mathcal{E}(\boldsymbol{r} t)$ happens to be the classical solution for the electric field.

Any state vector that obeys the relation (23) will also obey the adjoint relation

$$
\langle| E^{(-)}(\boldsymbol{r} t)=\mathcal{E}^{*}(\boldsymbol{r} t)\langle|
$$

Hence the $n$ th-order correlation function will indeed factorize into the form

$$
G^{(n)}\left(x_{1}, \ldots, x_{2 n}\right)=\mathcal{E}^{*}\left(x_{1}\right) \cdots \mathcal{E}^{*}\left(x_{n}\right) \mathcal{E}\left(x_{n+1}\right) \cdots \mathcal{E}\left(x_{2 n}\right)
$$

that we require for $n$ th-order coherence. Such states represent fully coherent fields, and delayed coincidence counting measurements carried out in them will reveal no photon correlations at all. To explain, for example, the Hanbury Brown-Twiss correlations we must use not pure coherent states but mixtures of them, for which the factorization conditions like Eq. (25) no longer hold. To see how these mixtures arise, it helps to discuss the modes of oscillation of the field individually.

The electromagnetic field in free space has a continuum of possible frequencies of oscillation, and a continuum of available modes of spatial oscillation at any given frequency. It is often simpler, instead of discussing all these modes at once, to isolate a single mode and discuss the behavior of that one alone. The field as a whole is then a sum of the contributions of the individual modes. In fact when experiments are carried out within reflecting enclosures the field modes form a discrete set, and their contributions are often physically separable.

The oscillations of a single mode of the field, as we have noted earlier, are essentially the same as those of a harmonic oscillator. The $n$th excitation state of the oscillator represents the presence of exactly $n$ light quanta in that mode. The operator that decreases the quantum number of the oscillator is usually written as $a$, and the adjoint operator-which raises the quantum number by one unit as $a^{\dagger}$. These operators then obey the relation

$$
a a^{\dagger}-a^{\dagger} a=1,
$$

which shows that their multiplication is not commutative. We can take the field operator $E^{(+)}(\boldsymbol{r} t)$ for the mode we are studying to be proportional to the operator $a$. Then any state vector for the mode that obeys the relation (23) will have the property

$$
a|\rangle=\alpha|\rangle,
$$

where $\alpha$ is some complex number. It is not difficult to solve for the state vectors that satisfy the relation (27) for any given value of $\alpha$. They can be expressed as a sum taken over all possible quantum-number states $|n\rangle$, $n=0,1,2, \ldots$ that takes the form

$$
|\alpha\rangle=e^{-(1 / 2)|\alpha|^{2}} \sum_{n=0}^{\infty} \frac{\alpha^{n}}{\sqrt{n !}}|n\rangle
$$

in which we have chosen to label the state with the arbitrary complex parameter $\alpha$. The states $|\alpha\rangle$ are fully coherent states of the field mode.

The squared moduli of the coefficients of the state $|n\rangle$ in Eq. (28) tell us the probability for the presence of $n$ quanta in the mode, and those numbers do indeed form a Poisson distribution, one with the mean value of $n$ equal to $|\alpha|^{2}$. The coherent states form a complete set of states in the sense that any state of the mode can be expressed as a suitable sum taken over them. As we have defined them they are equivalent to certain oscillator states defined by Schrödinger (1926) in his earliest discussions of wave functions. Known thus from the very beginning of wave mechanics, they seemed not to have found any important role in the earlier development of the theory.

Coherent excitations of fields have a particularly simple way of combining. Let us suppose that one excitation mechanism brings a field mode from its empty state $|0\rangle$ to the coherent state $\left|\alpha_{1}\right\rangle$. A second mechanism could bring it, for example, from the state $|0\rangle$ to the state $\left|\alpha_{2}\right\rangle$. If the two mechanisms act simultaneously the resulting state can be written as $e^{i \varphi}\left|\alpha_{1}+\alpha_{2}\right\rangle$ where $e^{i \varphi}$ is a phase factor that depends on $\alpha_{1}$ and $\alpha_{2}$, but we don't need to know it since it cancels out of the expression for the density operator

$$
\rho=\left|\alpha_{1}+\alpha_{2}\right\rangle\left\langle\alpha_{1}+\alpha_{2}\right| \text {. }
$$

This relation embodies the superposition principle for field excitations and tells us all about the resulting quan- 


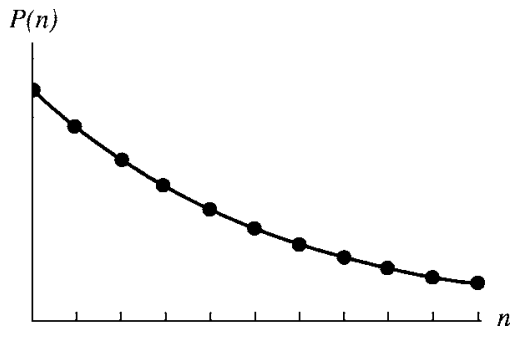

FIG. 6. Geometrical or fixed-ratio sequence of probabilities for the presence of $n$ quanta in a mode that is excited chaotically.

tum statistics. It is easily generalized to treat the superposition of many excitations. If, say, $j$ coherent excitations were present, we should have a density operator

$$
\rho=\left|\alpha_{1}+\cdots+\alpha_{j}\right\rangle\left\langle\alpha_{1}+\cdots+\alpha_{j}\right| .
$$

Let us suppose now that the individual excitation amplitudes $\alpha_{j}$ are in one or another sense random complex numbers. Then the sum $\alpha_{1}+\cdots+\alpha_{j}$ will describe a suitably defined random walk in the complex plane. In the limit $j \rightarrow \infty$ the probability distribution for the sum $\alpha=\alpha_{1}+\cdots+\alpha_{j}$ will be given by a Gaussian distribution which we can write as

$$
\mathrm{P}(\alpha)=\frac{1}{\pi\langle n\rangle} e^{-|\alpha|^{2} /\langle n\rangle},
$$

in which the mean value of $|\alpha|^{2}$, which has been written as $\langle n\rangle$, is the mean number of quanta in the mode.

The density operator that describes this sort of random excitation is a probabilistic mixture of coherent states,

$$
\rho=\frac{1}{\pi\langle n\rangle} \int e^{-|\alpha|^{2} /\langle n\rangle}|\alpha\rangle\langle\alpha| d^{2} \alpha,
$$

where $d^{2} \alpha$ is an element of area in the complex plane. When we express $\rho$ in terms of $m$-quantum states by using the expansion (28), we find

$$
\rho=\frac{1}{1+\langle n\rangle} \sum_{m=0}^{\infty}\left(\frac{\langle n\rangle}{1+\langle n\rangle}\right)^{m}|m\rangle\langle m| .
$$

This kind of random excitation mechanism is thus always associated with a geometrical or fixed-ratio distribution of quantum numbers (Fig. 6). In the best known example of the latter, the Planck distribution, we have $\langle n\rangle=\left(e^{h \nu / k T}-1\right)^{-1}$, and the density operator (33) then contains the familiar thermal weights $e^{-m h v / k T}$.

There is something remarkably universal about the geometrical sequence of $n$-quantum probabilities. The image of chaotic excitation we have derived it from, on the other hand, excitation in effect by a random collection of lasers, may well seem rather specialized. It may be useful therefore to have a more general way of characterizing the same distribution. If a quantum state is specified by the density operator $\rho$, we may associate with it an entropy $S$ given by

$$
S=-\operatorname{Tr}(\rho \ln \rho),
$$

which is a measure, roughly speaking, of the disorder characteristic of the state. The most disordered, or chaotic, state is reached by maximizing $S$, but in finding the maximum we must observe two constraints. The first is

$$
\operatorname{Tr} \rho=1,
$$

which says simply that all probabilities add up to 1 . The second is

$$
\operatorname{Tr}\left(\rho a^{\dagger} a\right)=\langle n\rangle,
$$

which fixes the average occupation number of the mode.

When $S$ is maximized, subject to these two constraints, we find indeed that the density operator $\rho$ takes the form given by Eq. (33). The geometrical distribution is thus uniquely representative of chaotic excitation. Most ordinary light sources consist of vast numbers of atoms radiating as nearly independently of one another as the field equations will permit. It should be no surprise then that these are largely maximum entropy or chaotic sources. When many modes are excited, the light they radiate is, in effect, colored noise and indistinguishable from appropriately filtered blackbody radiation.

For chaotic sources, the density operator (32) permits us to evaluate all of the higher-order correlation functions $G^{(n)}\left(x_{1}, \ldots, x_{2 n}\right)$. In fact they can all be reduced (Glauber, 1965) to sums of products of first-order correlation functions $G^{(1)}\left(x_{i} x_{j}\right)$. In particular, for example, the Hanbury Brown-Twiss coincidence rate corresponding to the two space-time points $x_{1}$ and $x_{2}$ can be written as

$$
\begin{aligned}
G^{(2)}\left(x_{1} x_{2} x_{2} x_{1}\right)= & G^{(1)}\left(x_{1} x_{1}\right) G^{(1)}\left(x_{2} x_{2}\right) \\
& +G^{(1)}\left(x_{1} x_{2}\right) G^{(1)}\left(x_{2} x_{1}\right) .
\end{aligned}
$$

The first of the two terms on the right side of this equation is simply the product of the two counting rates that would be measured at $x_{1}$ and $x_{2}$ independently. The second term is the additional delayed coincidence rate detected first by Hanbury Brown and Twiss, and it is indeed contributed by a two-photon interference effect. If we let $x_{1}=x_{2}$, which corresponds to zero time delay in their experiment, we see that

$$
G^{(2)}\left(x_{1} x_{1} x_{1} x_{1}\right)=2\left\{G^{(1)}\left(x_{1} x_{1}\right)\right\}^{2},
$$

or the coincidence rate for vanishing time delay should be double the background or accidental rate.

The Gaussian representation of the density operator in terms of coherent states is an example of a broader class of so-called "diagonal representations" that are quite convenient to use-when they are available. If the density operator for a single mode, for example, can be written in the form

$$
\rho=\int \mathrm{P}(\alpha)|\alpha\rangle\langle\alpha| d^{2} \alpha,
$$

then the expectation values of operator products like $a^{\dagger n} a^{m}$ can be evaluated as simple integrals over the function $\mathrm{P}$ such as 


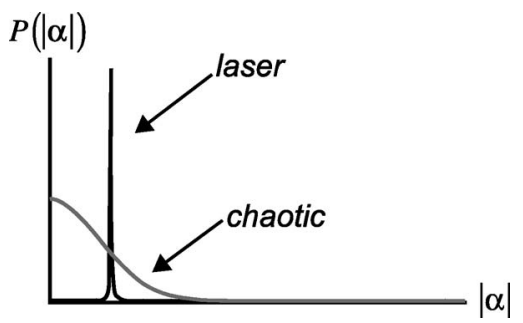

FIG. 7. The quasiprobability function $\mathrm{P}(|\alpha|)$ for a chaotic excitation is Gaussian in form, while for a stable laser beam it takes on nonzero values only near a fixed value of $|\alpha|$.

$$
\left\{a^{\dagger n} a^{m}\right\}_{\mathrm{av}}=\int \mathrm{P}(\alpha) \alpha^{* n} \alpha^{m} d^{2} \alpha .
$$

The function $\mathrm{P}(\alpha)$ then takes on some of the role of a probability density, but that can be a bit misleading since the condition that the probabilities derived from $\rho$ all be positive or zero does not require $\mathrm{P}(\alpha)$ to be positive definite. It can and sometimes does take on negative values over limited areas of the $\alpha$-plane in certain physical examples, and it may also be singular. It is a member, as we shall see, of a broader class of quasiprobability densities. The representation (39), and the Prepresentation, unfortunately is not always available (Sudarshan, 1963; Cahill and Glauber, 1969a, 1969b). It cannot be defined, for example, for the familiar "squeezed" states of the field in which one or the other of the complimentary uncertainties is smaller than that of the coherent states.

The difference between a monochromatic laser beam and a chaotic beam is most easily expressed in terms of the function $\mathrm{P}(\alpha)$. For a stationary laser beam the function $\mathrm{P}$ depends only on the magnitude of $\alpha$ and vanishes unless $|\alpha|$ assumes some fixed value. A graph of the function $\mathrm{P}$ is shown in Fig. 7, where it can be compared with the Gaussian function for the same mean occupation number $\langle n\rangle$ given by Eq. (31).

How do we measure the statistical properties of photon distributions? A relatively simple way is to place a photon counter in a light beam behind either a mechanical or an electrical shutter. If we open the shutter for a given length of time $t$, the counter will register some random number $n$ of photons. By repeating that measurement sufficiently many times we can establish a statistical distribution for those random integers $n$. The analysis necessary to derive this distribution mathemati-

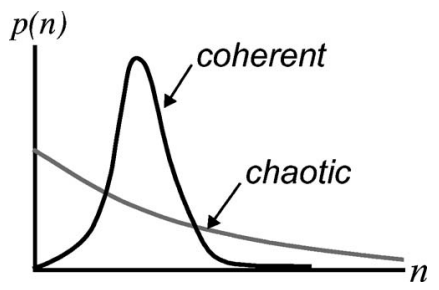

FIG. 8. The two $\mathrm{P}(|\alpha|)$ distributions of Fig. 7 lead to different photon occupation number distributions $p(n)$ : for chaotic excitation a geometric distribution, for coherent excitation a Poisson distribution. cally can be a bit complicated since it requires, in general, a knowledge of all higher-order correlation functions. Experimental measurements of the distribution, conversely, can tell us about those correlation functions.

For the two cases in which we already know all correlation functions, it is particularly easy to find the photocount distributions. If the average rate at which photons are recorded is $w$, then the mean number recorded in time $t$ is

$$
\langle n\rangle=w t
$$

In a coherent beam the result for the probability of $n$ photocounts is just the Poisson distribution

$$
p_{n}(t)=\frac{(w t)^{n}}{n !} e^{-w t}
$$

In a chaotic beam, on the other hand, the probability of counting $n$ quanta is given by the rather more spreadout distribution

$$
p_{n}(t)=\frac{1}{1+w t}\left(\frac{w t}{1+w t}\right)^{n} .
$$

These results, which are fairly obvious from the occupation number probabilities implicit in Eqs. (28) and (33), are illustrated in Fig. 8.

Here is a closely related question that can also be investigated experimentally without much difficulty: If we open the shutter in front of the counter at an arbitrary moment, some random interval of time will pass before the first photon is counted. What is the distribution of those random times? In a steady coherent beam, in fact, it is just an exponential distribution

$$
\mathrm{W}_{\mathrm{coh}}=w e^{-w t}
$$

while in a chaotic beam it assumes the less obvious form

$$
\mathrm{W}_{\mathrm{ch}}(t)=\frac{w}{(1+w t)^{2}} .
$$

There is an alternative way of finding a distribution of time intervals. Instead of simply opening a shutter at an arbitrary moment, we can begin the measurement with the registration of a given photocount at time zero and then ask what is the distribution, of time intervals until the next photocount. This distribution, which we may

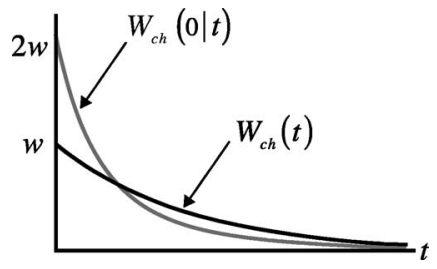

FIG. 9. Time interval distributions for counting experiments in a chaotically excited mode: $\mathrm{W}_{\mathrm{ch}}(t)$ is the distribution of intervals from an arbitrary moment until the first photocount. $\mathrm{W}_{\mathrm{ch}}(0 \mid t)$ is the distribution of intervals between two successive photocounts. 


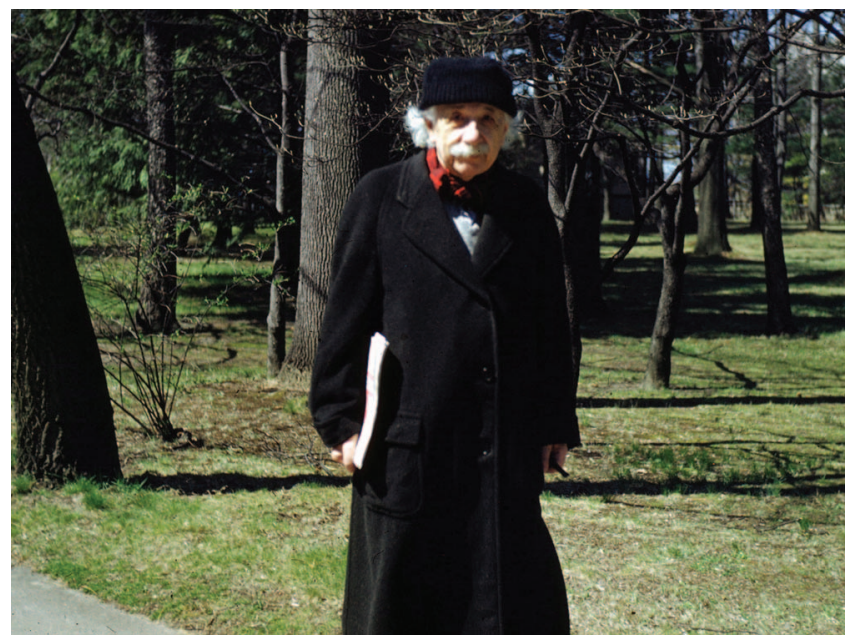

FIG. 10. (Color) Professor Einstein, encountered in the spring of 1951 in Princeton, NJ.

write as $\mathrm{W}(0 \mid t)$, takes the same form for a coherent beam as it does for the measurement described earlier, which starts at arbitrary moments,

$$
\mathrm{W}_{\text {coh }}(0 \mid t)=w e^{-w t}=\mathrm{W}_{\text {coh }}(t) .
$$

This identity is simply a restatement of the statistically independent or uncorrelated quality of all photons in a coherent beam.

For a chaotic beam, on the other hand, the distribution $\mathrm{W}_{\mathrm{ch}}(0 \mid t)$ takes a form quite different from $\mathrm{W}_{\mathrm{ch}}(t)$. It is

$$
\mathrm{W}_{\mathrm{ch}}(0 \mid t)=\frac{2 w}{(1+w t)^{3}},
$$

an expression which exceeds $\mathrm{W}_{\mathrm{ch}}(t)$ for times for which $w t<1$, and is in fact twice as large as $\mathrm{W}_{\mathrm{ch}}(t)$ for $t=0$ (Fig. $9)$. The reason for that lies in the Gaussian distribution of amplitudes implicit in Eqs. (31) and (32). The very fact that we have counted a photon at $t=0$ makes it more probable that the field amplitude $\alpha$ has fluctuated to a large value at that moment, and hence the probability for counting a second photon remains larger than average for some time later. The functions $\mathrm{W}_{\mathrm{ch}}(t)$ and $\mathrm{W}_{\mathrm{ch}}(0 \mid t)$ are compared in Fig. 8.

All of the experiments we have discussed thus far are based on the procedure of photon counting, whether with individual counters or with several of them arranged to be sensitive in delayed coincidence. The functions they measure, the correlation functions $G^{(n)}$, are all expectation values of products of field operators written in a particular order. If one reads from right to left, the annihilation operator always precedes the creation operators in our correlation functions, as they do, for example, in Eq. (19) for $G^{(2)}$. It is that so-called "normal ordering" that gives the coherent states, and the quasiprobability density $\mathrm{P}(\alpha)$ the special roles they occupy in discussing this class of experiments.

But there are other kinds of expectation values that one sometimes needs in order to discuss other classes of experiments. These could, for example, involve sym- metrically ordered sums of operator products, or even antinormally ordered products which are opposite to the normally ordered ones. The commutation relations for the multiplication of field operators will ultimately relate all these expectation values to one another, but it is often possible to find much simpler ways of evaluating them. There exists a quasiprobability density that plays much the same role for symmetrized products as the function $\mathrm{P}$ does for the normally ordered ones. It is, in fact, the function Wigner (1932) devised in 1932 as a kind of quantum mechanical replacement for the classical phase space density. For antinormally ordered operator products, the role of the quasiprobability density is taken over by the expectation value which for a single mode is $(1 / \pi)\langle\pi|\rho| \pi\rangle$. The three quasiprobability densities associated with the three operator orderings and whatever experiments they describe are all members of a larger family that can be shown to have many properties in common (Cahill and Glauber, 1969a, 1969b).

The developments I have described to you were all relatively early ones in the development of the field we now call quantum optics. The further developments that have come in rapid succession in recent years are too numerous to recount here. Let me just mention a few. A great variety of careful measurements of photon counting distributions and correlations of the type we have discussed have been carried out (Arecchi, 1969; Jakeman and Pike, 1969) and furnish clear agreement with the theory. They have furthermore shown in detail how the properties of laser beams change as they rise in power from below threshold to above it.

The fully quantum mechanical theory of the laser was difficult to develop (Scully and Lamb, 1967; Lax, 1968; Haken, 1970) since the laser is an intrinsically nonlinear device, but only through such a theory can its quantum noise properties be understood. The theories of a considerable assortment of other kinds of oscillators and amplifiers have now been worked out.

Nonlinear optics has furnished us with new classes of quantum phenomena such as parametric downconversion in which a single photon is split into a pair of highly correlated or entangled photons. Entanglement has been a rich source of the quantum phenomena that are perhaps most interesting — and baffling - in everyday terms.

It is worth emphasizing that the mathematical tools we have developed for dealing with light quanta can be applied equally well to the much broader class of particles obeying Bose-Einstein statistics. These include atoms of ${ }^{4} \mathrm{He},{ }^{23} \mathrm{Na},{ }^{87} \mathrm{Rb}$, and all of the others which have recently been Bose condensed by optical means. When proper account is taken of the atomic interactions and nonvanishing atomic masses, the coherent state formalism is found to furnish useful descriptions of the behavior of these bosonic gases.

The formalism seems likewise to apply to subatomic particles, to bosons that are only short-lived. Pions that emerge by hundreds or even thousands from the highenergy collisions of heavy ions are also bosons. Pions of similar charge have a clearly noticeable tendency to be 
emitted with closely correlated momenta, an effect which is evidently analogous to the Hanbury BrownTwiss correlation of photons, and invites the same sort of analysis (Glauber, 2006).

Particles obeying Fermi-Dirac statistics, of course, behave quite differently from photons or pions. No more than a single one of them ever occupies any given quantum state. This kind of reckoning associated with fermion fields is radically different therefore from the sort we have associated with bosons, like photons. It has proved possible, nonetheless, to develop an algebraic scheme (Cahill and Glauber, 1999) for calculating expectation values of products of fermion fields that is remarkably parallel to the one we have described for photon fields. There is a one-to-one correspondence between the mathematical operations and expressions for boson fields, on the one hand, and fermion fields, on the other hand. That correspondence has promise of proving useful in describing the dynamics of degenerate fermion gases.

I'd like, as a final note, to share with you an experience I had in 1951, while I was a postdoc at the Institute for Advanced Study in Princeton. Possessed by the habit of working late at night-in fact on photon statistics (Glauber, 1951) at the time-I didn't often appear at my desk early in the day. Occasionally I walked out to the Institute around noon, and that was closer to the end of the work day for Professor Einstein. Our paths thus crossed quite a few times, and on one of those occasions I had ventured to bring my camera. He seemed more than willing to let me take his picture as if acknowledging his role as a local landmark, and he stood for me just as rigidly still. Here, in Fig. 10, is the hitherto unpublished result. I shall always treasure that image, and harbor the enduring wish I had been able to ask him just a few questions about that remarkable year, 1905.

\section{REFERENCES}

Arecchi, F. T., 1969, in Quantum Optics, Course XLII, Enrico Fermi School, Varenna, 1969, edited by R. J. Glauber (Academic Press, New York).
Born, M., and E. Wolf, 1959, Principles of Optics (Pergamon Press, London), Chap. X.

Cahill, K. E., and R. J. Glauber, 1969a, Phys. Rev. 177, 1857.

Cahill, K. E., and R. J. Glauber, 1969b, Phys. Rev. 177, 1882.

Cahill, K. E., and R. J. Glauber, 1999, Phys. Rev. A 59, 1538.

Dirac, P. A. M., 1927a, Proc. R. Soc. London 114, 243.

Dirac, P. A. M., 1927b, Proc. R. Soc. London 114, 710.

Dirac, P. A. M., 1958, The Principles of Quantum Mechanics, 4th ed. (Oxford University Press), p. 9.

Einstein, A., 1905, Ann. Phys. 17, 132.

Glauber, R. J., 2006, Quantum Optics and Heavy Ion Physics, e-print org/nucl-th/0604021.

Glauber, R. J., 1951, Phys. Rev. 84, 395.

Glauber, R. J., 1965, in Quantum Optics and Electronics (Les Houches 1964), edited by C. de Witt, A. Blandin, and C. Cohen-Tannoudji (Gordon and Breach, New York), p. 63.

Haken, H., 1970, in Encyclopedia of Physics XXV/2c, edited by S. Flügge (Springer-Verlag, Heidelberg).

Hanbury Brown, R., and R. Q. Twiss, 1954, Philos. Mag. 45, 663.

Hanbury Brown, R., and R. Q. Twiss, 1956, Nature (London) 177, 27.

Hanbury Brown, R., and R. Q. Twiss, 1957a, Proc. R. Soc. London, Ser. A 242, 300.

Hanbury Brown, R., and R. Q. Twiss, 1957b, Proc. R. Soc. London, Ser. A 243, 29.

Jakeman, E., and E. R. Pike, 1969, J. Phys. A 2, 411.

Javan, A., W. R. Bennett, and D. R. Herriot, 1961, Phys. Rev. Lett. 6, 106.

Lax, M., 1968, in Brandeis University Summer Institute Lectures (1966), Vol. II, edited by M. Cretien et al. (Gordon and Breach, New York).

Planck, M., 1900a, Ann. Phys. 306, 69.

Planck, M., 1900b, Ann. Phys. 306, 719.

Purcell, E. M., 1956, Nature (London) 178, 1449.

Rebka, G. A., and R. V. Pound, 1957, Nature (London) 180, 1035.

Scarl, D. B., 1968, Phys. Rev. 175, 1661.

Schrödinger, E., 1926, Naturwiss. 14, 664.

Scully, M. O., and W. E. Lamb, Jr., 1967, Phys. Rev. 159, 208.

Sudarshan, E. C. G., 1963, Phys. Rev. Lett. 10, 277.

Titulaer, U. M., and R. J. Glauber, 1965, Phys. Rev. 140, B676. Titulaer, U. M., and R. J. Glauber, 1966, Phys. Rev. 145, 1041. Wigner, E., 1932, Phys. Rev. 40, 749. 